\title{
Riding Through Walls: A journey of physical computing through Google Street View
}

\author{
Megan L. Smith \\ University of Regina \\ Regina, SK, Canada \\ megan.smith@uregina.ca
}

\author{
John Desnoyers-Stewart \\ Simon Fraser University \\ Surrey, BC, Canada \\ desnoyer@sfu.ca
}

\begin{abstract}
Over two years, artist and researcher Dr. Megan Smith cycled through image tile after image tile of the Google Street View platform, physically working her way across $6300 \mathrm{~km}$ of highwayredefining what the great Canadian road trip can be. Starting out with a goal to network a stationary bike with the internet, along the way she dropped into an other experience: a space of physical glitches, a jaunt through other people's histories, a glimpse into the lives of the people who drive the Google-Mobiles-their pit-stops, lunches, and road kill. It was a digitally enhanced life experience far beyond what the step counter on a fitbit can offer. It was not an 'Everyware,' but instead an intimate glimpse into the layers of preserved digital visual culture along a focused route that revealed human and algorithmic errors hidden deep within the structure of the amazing archive of Google data. Each blurred statue and virtual dead-end hinted at the infrastructure and labour, which has produced a wealth of information hidden beyond the boundaries of the publicly accessible platform. Perpetually triggered by human force, it was an experience of places beyond reach that were nonetheless felt, seen, and discovered through a compulsive drive to push through the Internet. The journey was filled with surprise and discovery, from the sudden appearance of angelic forms on the screen, to messages of affection scrawled on the rocks of the endless Canadian Shield. These moments of excitement punctuated the endless hours of cycling, which required both physical endurance and mental perseverance. While most forays into Google Street View last a few minutes and involve magical teleportation from one landmark to the next, Smith pedalled for a total of $\mathbf{3 3 7}$ hours. This duration almost exactly matches Google's prediction for the over $6300 \mathrm{~km}$ journey, demonstrating the real physicality of her virtual endeavour, and the detailed accuracy of the geo-data within the platform. From coast to coast, Smith was joined physically and virtually by supporters who rode alongside or cheered as she achieved milestone after milestone, getting ever nearer to Canada's east coast. The entire adventure was live cast and documented publicly on social media. An archive of the performance can be found across Twitter, Facebook, Instagram and YouTube along with directions to build a DIY networked bike on Instructables. Through a series of events and workshops the project engaged the community and spawned interest in virtual road tripping and exploration. This paper will discuss the sights and experiences encountered along this journey across Canada, and what those experiences say about digitally experiencing geography and culture through a visual archive: the timelessness of experiences frozen in the database, the limitations of human error within a platform that is managed by algorithms, and what it really means to be physically computing.
\end{abstract}

Physical computing. Google Street View. Maker culture. Virtual road trip. Networked bike.

\section{INTRODUCTION}

Over a two-year period, artist Megan Smith cycled across Canada virtually through Google Street View. She pedalled her networked stationary bike through image tile after image tile along the transCanada highway. Navigating this path enabled a physical encounter with a digital experience, a chronicling of a research path through a mapping tool, and carved out a new space for research in physical computing. The project was live cast and archived on YouTube, and shared across social media, with a photo series published on Instagram under the hashtag \#ridingthroughwalls. On two occasions the artist performed publicly and she delivered a series of workshops teaching participants how to build their own networked bikes. In this paper, we will unpack some of the discoveries of the journey as well as attempt to articulate the process and experience of physically computing through the Internet. This researchcreation project explored Google's vast archive of data on their Street View platform via a digital expedition that humanised the system through the familiar narrative of a road trip. 
In its attempt to make just about anywhere accessible just about anywhere else, Google Street View is what Greenfield (2006) calls an everyware. As an everyware, it attempts to colonise everyday life (Greenfield 2006), altering the way in which we experience the world and allowing instantaneous transportation between destinations around the world; however, Smith's journey confronts the pervasive everyware of this technology, benefiting from its capacity to connect her to anywhere while simultaneously adhering to a real, physical path and experiencing the technology over a real, physical period of time.

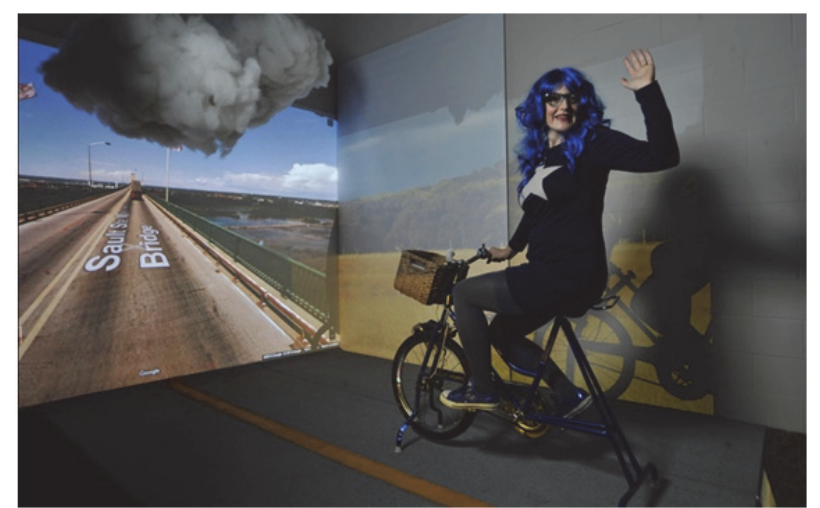

Figure 1: Megan Smith ready to ride through walls on the networked bike set up in her studio.

\section{THE CONCEPT AND DESIGN}

Smith initially started working with dynamos and small generators in an effort to build an interactive bicycle that could generate power to run a projector with the goal of building a mobile photobombing project. Her established art practice in mapping, GPS, and social media storytelling soon moved the bicycle project towards the creation of an installation that could physically push through the internet, specifically through Google Maps' feature application, Google Street View, a platform that virtually represents the globe in a network of interconnected $360^{\circ}$ images. This platform is built through stitching together of 'millions of panoramic images' collected primarily by Google's familiar fleet of specialised cars (Google 2019).

The concept of building a bike to ride through Google Street View is nearly as old as the platform itself. In 2009, Aki Mimoto created what he called an "unfinished, unstable prototype" using Vuzix VR that allowed him to ride through Street View on his bike trainer (Mimoto 2009). In 2012, Jeffery Adkins published instructions on how to use an Arduino microcontroller to connect the pedalling of a stationary bike to Street View on Low End Mac. He used a reed switch and magnet to detect the crank revolutions and then send an up arrow keyboard command at regular intervals (Adkins 2012).
In 2014, Smith, in collaboration with Krazatchu Design Systems developed a Street View bike installation for Nuit Blanche Ottawa + Gatineau. This version included a web app and showed the rider's progress graphically on the screen. For Riding Through Walls, the final iteration of this project, funded by the Social Science and Humanities Research Council of Canada, Smith reverted to a simpler design in order to build a project with a stable and accessible educational workshop component that would attract beginner makers and children to learn about code and electronics. Re-working Adkins original code, Smith added two buttons to steer left and right, facilitating the navigation of Street View for the long journey ahead.

In mid-2015, the project took physical form as an installation in the artist's studio at the University of Regina, SK, Canada. The artist constructed an artificial road and grass verge within her studio and hung fabricated clouds within the scene. On the road was the vintage Air Wing stationary bike, revived from the recesses of a garage where it had been left unused for many years. The vintage Air Wing was chosen for the journey for its aesthetic charm that contrasted with the high-tech system it was ridden through.

The bicycle was equipped with an Arduino Leonardo microcontroller hidden within the bicycle's basket, a magnet attached to the wheel and a corresponding reed switch on the frame, and two buttons affixed to the bicycle handlebars. This electronic system connected via USB to a computer. A projector was used to fill the wall with the Google Street View content in front of the artist, aligning with the artificial road and immersing her into the life-sized presentation of the road ahead. A second perpendicular projection displayed an image, such as a local landmark or scene, which characterised each particular stretch of the journey. Two moveable clouds made of polyester batting were hung from the ceiling to complete the scene. The wiring schematic of the implementation for Riding Through Walls is shown in figure 2 and detailed instructions including video for building a bike are posted on Instructables.com:

https://www.instructables.com/id/Riding-ThroughWalls-Google-Street-View-Stationary/ 


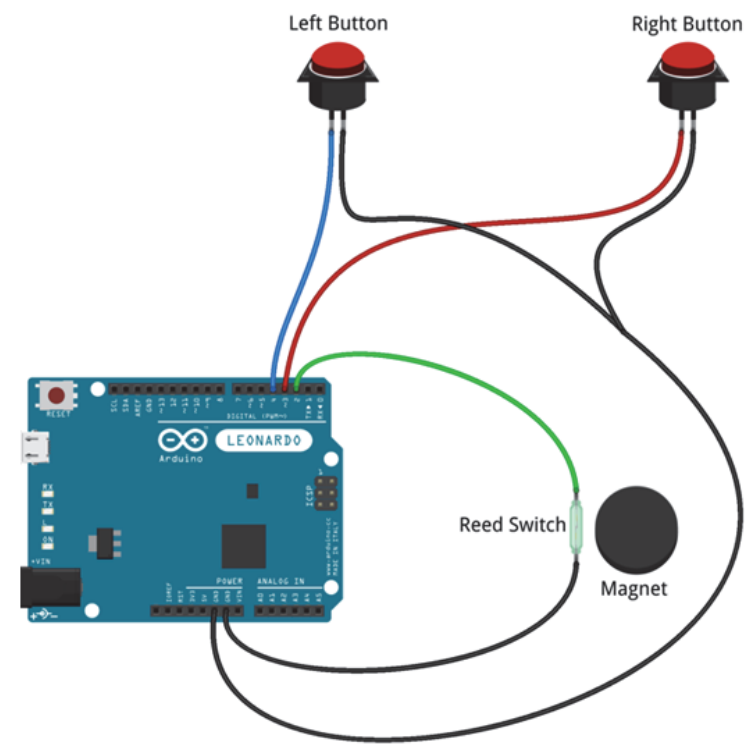

fritzing

Figure 2: The wiring schematic for the networked bike.

The simplicity of this implementation was a critical element of the project as it was designed to incorporate affordable, off the shelf electronics, to make the project feasible for schools and the community at large. The components can be easily purchased for approximately $\$ 40$ USD. As Smith was riding across the country, a commercial system for riding a bike in Street View, BikeAround, was released. This tool is designed for clinical settings to help patients with cognitive and physical disabilities in their rehabilitation, while simultaneously enabling a mechanism to trigger memories and aid with recollection. While it features a refined setup and smoother transitions between images, BikeAround costs $\$ 14,500$ USD (Larkin 2018). Although useful in clinical settings, this price makes it impractical for the majority of users to experience and underlies the value of maker culture in giving a wider population access to this kind of technology.

\section{THE JOURNEY \& PERFORMANCE}

The ride routinely began in the studio with a ritual of repetitive tasks that setup the documentation of the performance and tested for technical issues: turn on the webcam and projector, match the starting location on Street View to the end of the last ride, input the ride into YouTube and activate the live stream, find a water bottle and place it in the basket, test the directional buttons on the handlebars, and finally, mount the bike and don headphones to listen to yet another audiobook, podcast, Spotify Playlist or just noise cancellation to drown out the noise of the pedalling. This was the IRL experience of each leg of the journey. The virtual experience was different.
The virtual journey began near the Mile 0 marker of the Trans-Canada Highway, in Victoria, British Columbia, looking out at the ocean and wondering what the man sitting on the park bench was thinking of the view, and whether he still returned to this spot on the day the virtual ride began. Google had captured him on their cameras in April 2014, but Smith was there on December 1, 2015. After gazing at the ocean view and the man, Smith turned east and began pedalling towards Cape Breton. It took over two years to reach her destination as the durational performance occurred on an intermittent schedule.

The cycle ride through Google Street View covered approximately $6,420 \mathrm{~km}$ spread across numerous individual rides that each averaged about 2 hours per ride, totalling 337 hours of physical engagement with the digital platform over the entire performance, a duration that closely matches Google's prediction for the journey by bike.

The Trans-Canada Highway was the primary route, though on occasion the artist took alternative routes recommended to her through audience participation, such as a Facebook vote that determined a deviation from the most efficient route at Hope, BC, to take a more scenic route up Highway 1 via Lytton. She also cycled along diverted streets and dirt roads when roadwork impeded access to the Trans-Canada Highway. Most interesting were the diversions around invisible barriers caused by gaps in the Google Street View data of unmapped terrain, or flawed image synching within the platform. The glitches in the platform led to moments of discovery of the human-in-the-loop within Google's seemingly autonomous system, such as occasions when the Google car is markedly pulled off the side of the road for a rest break or perhaps to change the system's hard drives.

At regular intervals the artist would stop to photograph landmarks, sites recommended to her by her social media followers, places that caught her attention, or arrested her for a moment of reflection, just as, she posits, any traveller might on a Great Canadian Road Trip. These photographs formed the Instagram image series and were later imprinted onto kitsch tourist ephemera such as coffee mugs and coasters. The experience of travelling along kilometres of highway was different though for Smith, as she was piercing through data that simulated reality. The visual stream formed a hyperreal digital experience that diverged from that of everyday physical reality.

The map below details the path, which the journey charted across Canada. It shows the two and a half year timespan of the expedition as a gradient stretching from coast to coast. The meandering line 


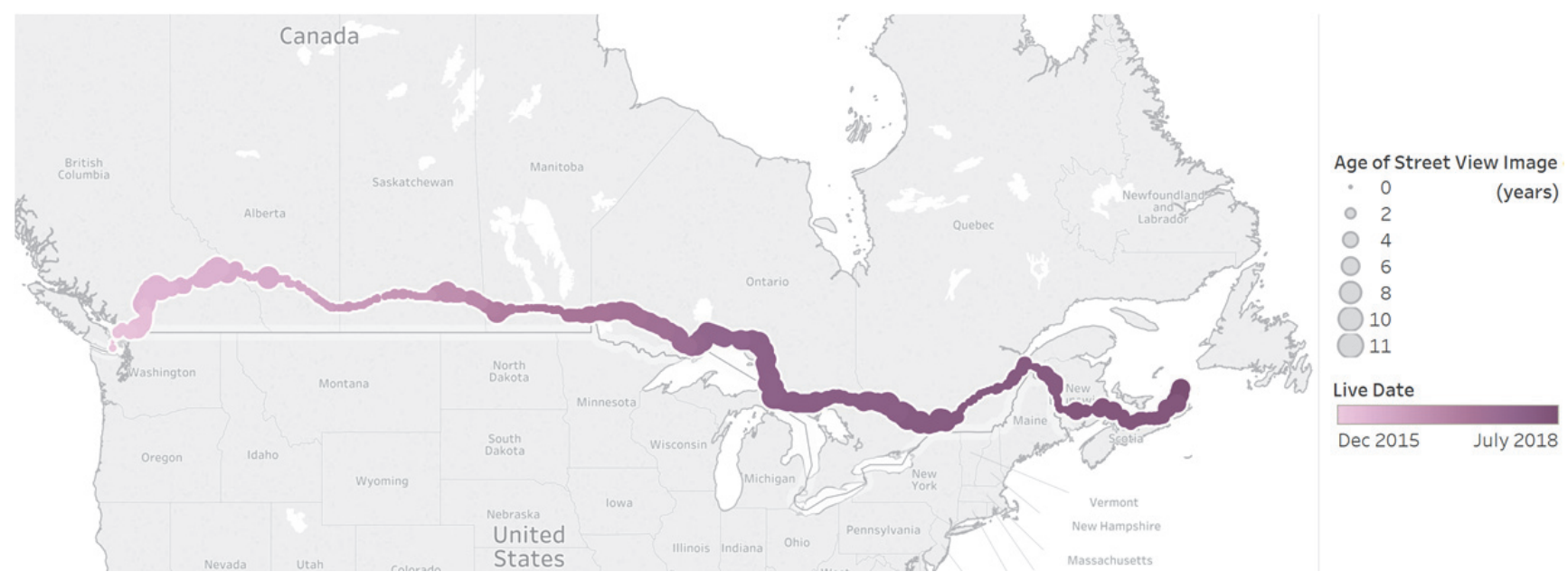

Figure 3: Map of the journey through Google Street View showing the age of street view images encountered (size) and live date (colour).

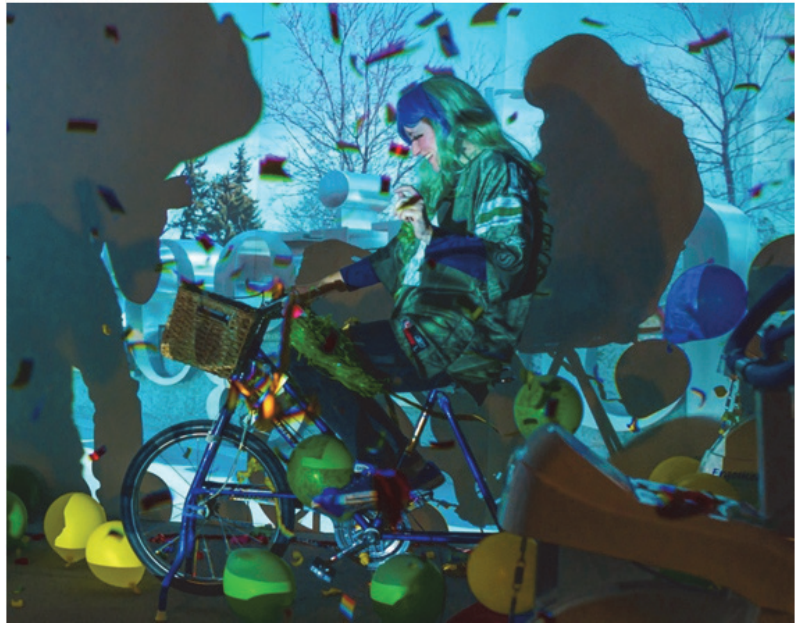

Figure 4: Photo of Moose Jaw to Regina Performance

is made up of circles representing each geocoded point where Smith documented her journey on Instagram. The size of each circle varies based on the age of the Street View image at the time when Smith cycled through that area, with the largest circles representing the oldest imagery. A map linking to image can be accessed here: Website: http://ridingthroughwalls.megansmith.ca/

Each leg of the journey was captured using a webcam, enabling all the rides to be recorded and broadcast on YouTube. While this was done to mimic the reciprocal perpetual logging and layering documentation of Google's systems, it also added a meta-layer to Smith's arrival into the city of Regina. The coinciding real and virtual layers were marked by a public performance where Smith cycled from Moose Jaw to Regina. During this 6hour window, the community of Regina who came to join the ride with Smith felt an excitement to take part in the blending of the virtual and the physical. The Canadian Broadcasting Corporation as well other local media were onsite to chronicle, live on the air, the arrival into the city. At the point when Smith rode through Regina, she and those listening on the radio, following on the livecast, or keeping pace with the performance through social media were traversing virtual space that she and fellow riders were simultaneously embodying in the physical. The moment was celebrated in an explosive culmination of confetti cannons and balloons dropping. The experience can be seen on YouTube:

https://www.youtube.com/channel/UCi8 Zt2A4BW wjCFwQNbiiFw

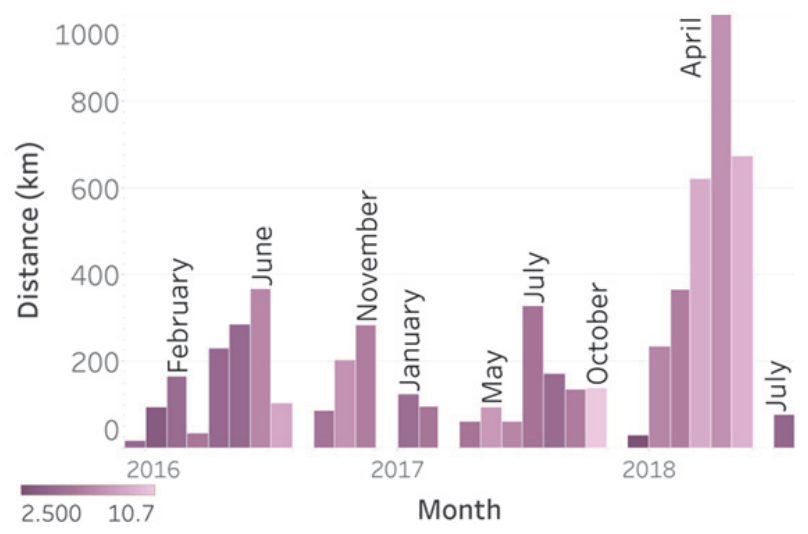

Figure 5: Distance covered per month. Colour shows average distance between images ( $\mathrm{km} / \mathrm{image})$.

Figure 5 charts Smith's progress across Canada and the frequency of image taking. Gaps can be seen when no riding occurred and a marked peak occurred within the final months of the performance. The photo documentation was sparsest in October 2017 after months of cycling across the endless Canadian Shield but subsequently peaked when Smith reached Thunder Bay, Ontario, where, just past the halfway point, Smith's pace rapidly accelerated towards the finish line. The installation moved from Smith's studio in May 2018 to be included in the URL:IRL exhibition. 
There, Smith finally rode to the East Coast destination during a public performance culminating the journey at the Dunlop Art Gallery in July 2018.

\section{THE DIGITAL EXPERIENCE}

The performance yielded visual experiences that would have been impossible on an actual ridesuch as the very real experience of falling through a bridge to the road below due to the platform's misunderstanding of an overpass as an intersection, which forced her route from one plane to another. These digital anomalies, or artefacts of the constructed experience, differed from what would be considered a normal ride across Canada, forming valuable insights through a new way of seeing the mapped canvas of a digitised world. Yet, the artist retained the sense that she was riding the distance, seeing the country, and partaking in a physical act of traversing landscape.

The artist was regularly transported from season to season as she seamlessly transitioned between trips of the Google-Mobile. Some stretches of highway were still relics of Google's first foray into Street View from 2007, while others were captured since Smith had embarked on her journey, a reminder of Google's continuous endeavour to maintain the database at a pace faster than her own journey through it.

Google's marketed vision of Street View as a way of landmark-hopping from the Eiffel Tower to the Pyramids to the Great Wall of China risks devaluing the spaces in between. Through this journey, the value of local landmarks is brought back into the forefront. Just as with an actual road trip, each passing artefact is bestowed with significance as it breaks up the occasionally monotonous journey. From graffiti on the Canadian Shield to local "World's Largest" statues, engaging with a virtual system meant for rapid consumption at a real-life pace allowed a rich understanding of such elements' significance in everyday life, beyond the hyperreal gaze of Google's maps and imagery.

In his photo essay The Nine Eyes of Google Street View, artist Jon Rafman claims that Street View captures reality with indifference, enabling moments to be captured without the intent of the human photographer and deferring the act of framing to the viewer (Rafman 2009). On her journey through Street View, this indifferent chain of imagery becomes Smith's very real experience as those moments unfold in real time, enabling them to regain the significance and spontaneity unwittingly captured in the database.

\subsection{Revealing Agency through Glitches}

Furthermore, Smith discovered moments in Street View that were cues to human decisions within their archive. These occurrences contradict the system's seeming mechanical indifference, as highlighted by Rafman, with hints of human agency that expose the pragmatic and aesthetic decisions made by the driver. While the system is designed to "avoid gaps in the 360 photos" and "create smooth transitions" (Google 2019), it does not account for drivers pulling off to the side of the road. So, on many occasions along the TransCanada, Smith was diverted onto the gravel, or the verge of small side roads, for brief rests among the wildflowers. These could be seen as non-spaces of the platform. They don't mesh with the supposedly perfect grid system of documenting the routes, but are clear places where the photographers made the choice to break off from the otherwise fixed trajectory. Analysts could potentially remove these human moments from the app by blending another year's photos into the diverted space, but they are left within the platform as Easter Eggs, surprises sometimes purposely placed within computer software for the dedicated user to discover.

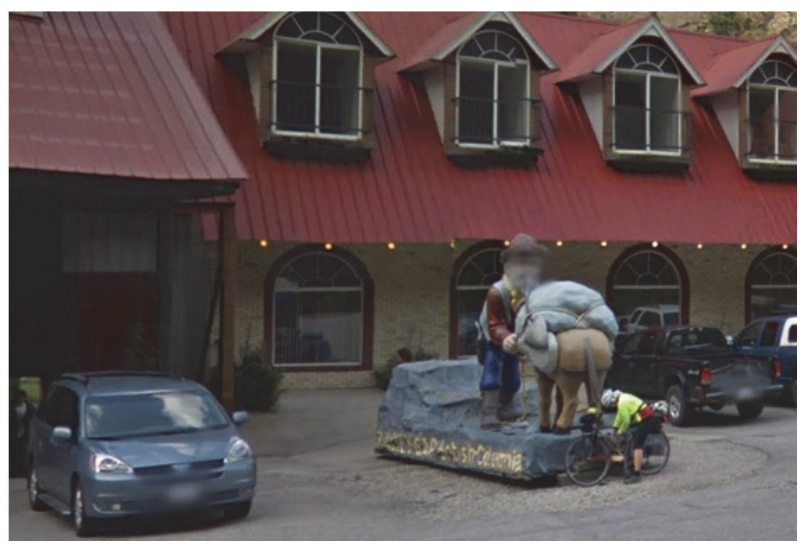

Figure 6: Blurred face of a statue. Image Captured from Google Street View (c) 2018 Google.

\subsubsection{Censored Statues}

Smith also believes that while the algorithm developed by Google to blur people's faces, license plates, and other identifying markers (Google 2019) does a basic job, to be fully effective it sometimes requires an analyst's human touch in postproduction. She occasionally saw faces that were not blurred, often in the form of hitch hikers who are markedly outside of the normal human populated spaces. Smith also found that public sculptures (Figure 6) were sometimes blurred, despite the fact that the images were clearly cartoon-like by nature. This was perceived as another indicator of purposeful intent, or agency, of the content builders, and that the creativity of the Google Street View platform is shared between the algorithm, the car driver/photographer, and the analyst. 
While the typically brief activity of visiting a landmark or one's home town in Google Street View tends to be a seamless experience, a durational engagement with the platform reveals an abundance of glitches produced by human and algorithmic actors. Pushing the platform outside of its intended design leads to results that lay bare the agency of both human and artificial intelligences, particularly where disparate intentions clash at the interface between the two.

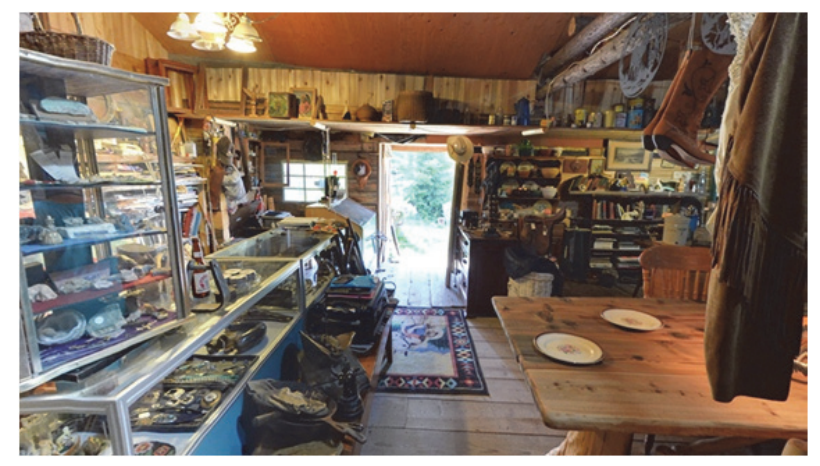

Figure 7: Unexpectedly riding through walls and ending up inside a building. Image Captured from Google Street View (c) 2014 Google.

One significant moment pertaining to this discovery was Smith's ride through a rural area of Northern British Columbia, when she was suddenly diverted from the highway into a roadside store where she found herself surrounded by handmade wood furniture and a hodgepodge of trinkets. This "virtual tour," a contribution welcomed by Google as a way to promote one's business on their platform, (Google 2019) had become inadvertently connected to the highway and sent Smith on a jarring, unexpected adventure. This anomaly has since been rectified in 2018 but can still be revisited by returning to an older version of the street view.

\subsubsection{Warped Infrastructure}

A recurring glitch throughout the journey was the warping of overhead infrastructure including overpasses, bridges, and signs. While the $360^{\circ}$ image stitching algorithm seemed to perform well with structures near the horizon, as objects passed overhead straight lines became bent and skewed by the system's inability to compensate for the cameras mismatched perspectives. This artefact was particularly noticeable and recurrently unsettling as these rigid structures burst apart and then reformed as they passed overhead.

These glitches become embedded within the architecture of Street View-just as consistently as it produces the intended functionality, so too it creates unexpected results missed during software testing. Any software project of such scale is bound to contain imperfections that don't necessarily interfere with regular use. The debugging process eliminates many of these 'flaws,' but nonetheless they persist, their rarity making them all that much more exciting to discover. Considering the scale of Google Street View it's possible, if not probable that Smith was the first to move between many of the automatically connected images, allowing her to uncover unique artefacts for the first time. As Smith claimed writing about Riding Through Walls part way through the journey:

"In this sense, post-internet art uses the social web as both a tool and a source of inspiration. This leads to reimagining situations, uncovering narratives, and the creation of new works of art shaped by tool and culture." (2018, p.105)

The glitches shaped the performance and influenced aesthetic choices in the documentation process, but most importantly they clearly identified the performance as a physical intervention into the digital infrastructure.
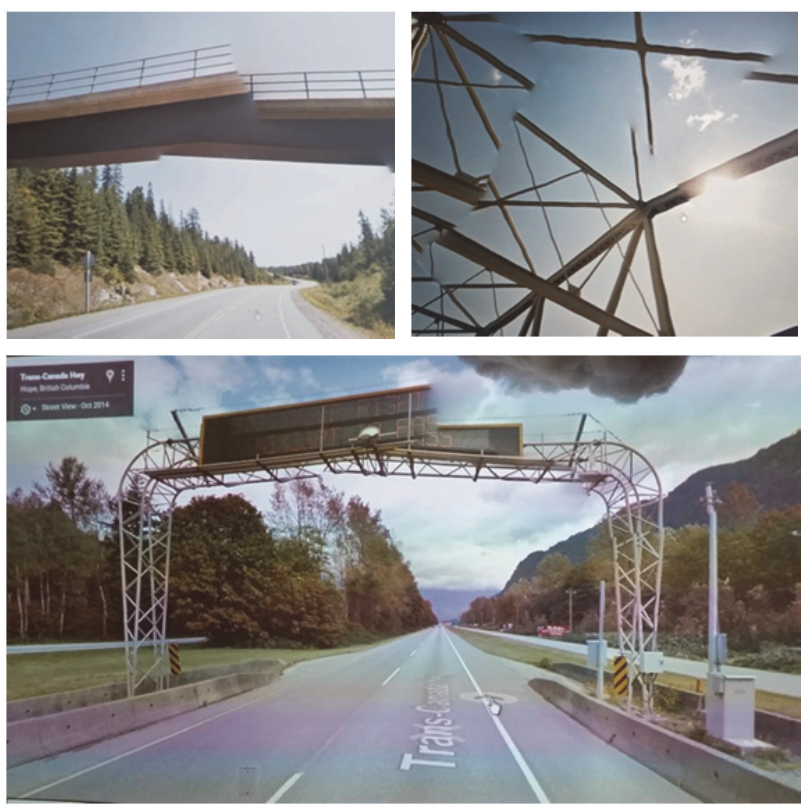

Figure 8: Warped structures. Image Captured from Google Street View (c) 2016-2018 Google.

\subsection{People}

Smith's documentation of her journey in Instagram snapshots follows a methodology, which continues the tradition of capturing the experience of a road trip through a selective but spontaneous act in the digital domain. This act of archiving serves to realise the significance of the people and places captured by Street View as more than just pixels in a digital archive. Rafman states that his work, Sixteen Google Street Views, "presented individuals... so that they resist becoming solely objects of the robotic gaze of an automated camera... [highlighting] the significance and uniqueness of the individual" (Rafman \& Steinman 2016, p.76). Similarly, Smith seeks to find unique and surprising elements among the endless database of imagery. 


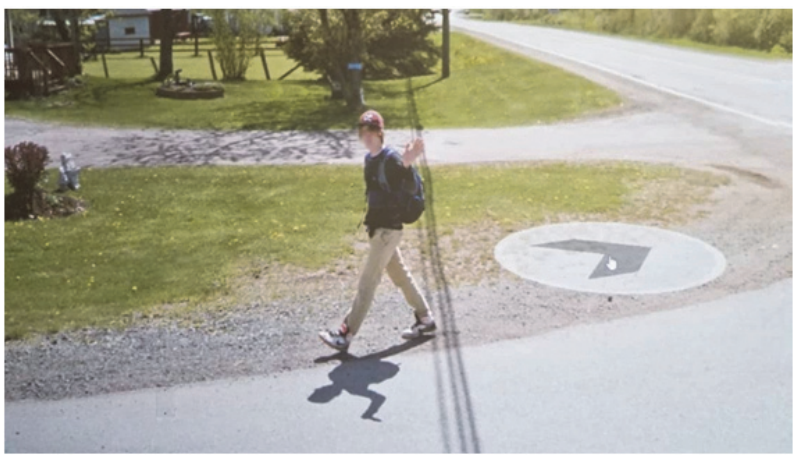

Figure 9: Smiling and waving was a common response to the Google-mobile's presence. Image Captured from Google Street View (c) 2018 Google.

Many people engaged with the Google car through waving at the cameras, as discussed by Smith in Anywhere (2018). In the YouTube archive, Smith can be seen waving back at these people, stopping to get a closer look at them, and then framing an image of the person. In some instances not all the angles of the Google cameras are blurred within the system architecture and clear images of the individual faces can be seen. On each occasion the artist tried to give the person some additional time on the livestream, as their acts affected the Google archive by demanding presence. In a sense, these people broke the fourth wall of the system and allowed for another crack of humanity within the structure.

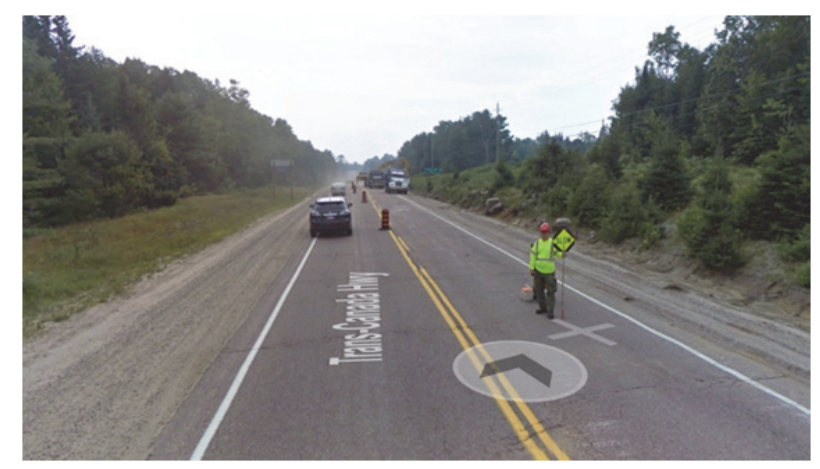

Figure 10: Megan Smith's Uncle in August 2009. Image Captured from Google Street View @ 2017 Google.

Other significant moments were when audience members would reach out to the artist to see if they had travelled by their homes or seen their family member. In Fig 10, the artist used Google Time machine to travel back to 2009, when her uncle was on a road crew near Petawawa, Ontario. She was then able to cycle by him and include his memory, and presence into the project.

The act of photographing the road trip through the digital space creates a unique opportunity to capture the plethora of beauty, human moments, memories, and wonder that can be found in the spaces in-between.

\subsection{Movement and Physicality}

This singular physical trajectory through the archive presents a small sample of many overlapping stories while also realizing the physicality of the data, which Google has amassed. Imagery captured as the artist passed from one tile to the next act as detectors of detail that is often invisible-the architecture of the system. Elements of the complex mechanical and digital structure that supports Google Street View were revealed through Smith's motion through the platform. This is seen in the blur in Figure 8, where the image appears to have a filter over it, but is in fact a space holder for the future image.

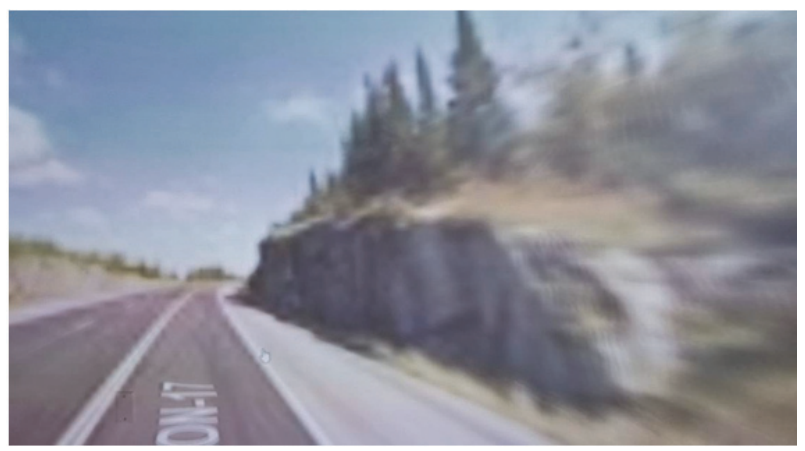

Figure 11: Between frames in Street View. Image Captured from Google Street View (c) 2017 Google.

\subsubsection{Copyrighted Landscapes}

Movement also delivered layers of branding and the strategies that Google uses to incorporate their logo into each image, each tile, and within each transition between image tiles. When bandwidth was slow the frame refresh rate lagged and this is when the extent of their branding was revealed. Figure 9 demonstrates the watermarked logo on the architecture between the image tiles, which is occasionally briefly seen, but a more detailed inspection of each image revealed an endless sea of watermarks cast upon these public spaces.

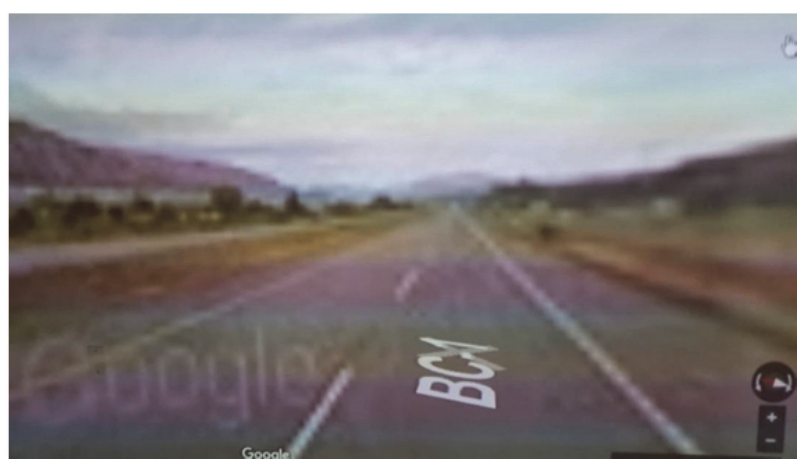

Figure 12: Google's copyright mark (bottom left) visible on each image tile. Image Captured from Google Street View @ 2018 Google. 


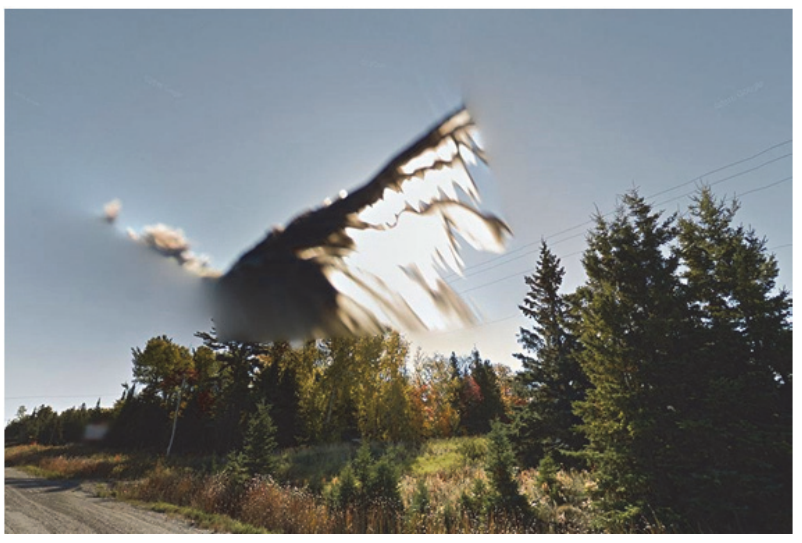

Figure 13: Bird-angel. Image Captured from Google Street View (C) 2018 Google.

\section{CONCLUSION}

As with a road trip, this content is but a small sample of the actual physical journey. The 10 images presented here were selected from 948 snapshots which Smith captured and posted to Instagram on her journey, yet those represent but a fraction of the over six-hundred thousand $360^{\circ}$ images she traversed. Among the relics of the performance archive, it is these Instagram images that relay the enhanced experience in detail. They acted to map her own course through the platform, are indicators of audience participation, and they enable her to relay a critical lens on the architecture of the platform.

While attempting to physically push through the Internet, Smith gradually performed a digitally enhanced experience. Riding Through Walls enabled Smith, a mother and professor, to accomplish the impossible-riding across the country without leaving her family or academic responsibilities behind. The physical engagement with audiences throughout the expedition enabled her to build into the project a rich narrative of her own discoveries, while embedding memories and desires of others into the performance. In travelling the Trans-Canada Highway, Smith encountered surprises and discoveries that revealed the human and algorithmic agency through nuances within the Google Street View platform. At the architectural level, these are the decisions of the photographers and analysts in the creation of content combined with the unexpected glitches embedded within the system's complex algorithms. Smith recorded these discoveries in the form of a public archive of her expedition. In accordance with her practice she worked to tell a new locative narrative, one that grappled with the megalith of the Google's mapping of the globe through the humanizing pastime of the road-trip.

The image in Figure 13, "Bird-angel," perhaps sums up the surprise and discovery of Smith's journey in a single photograph. What was in actuality the untimely demise of an innocent bird, became transformed into an angelic apparition. An ordinary event made hyperreal through the physical penetration of the digital archive.

\section{ACKNOWLEDGEMENTS}

This research was supported by the Social Sciences and Humanities Research Council of Canada Insight Development Grant.

\section{REFERENCES}

Adkins, J. (2012) Bike Across the Country Without Leaving Home. http://lowendmac.com/2012/bikeacross-the-country-without-leaving-home/ (retrieved 11 March 2019).

Google (2019) Google Street View. https://www.google.com/streetview (retrieved 11 March 2019).

Greenfield, A. (2006) Everyware: The Dawning Age of Ubiquitous Computing. New Riders Publishing, Berkeley, CA.

Larkin, M. (2018). BikeAround: Making memories accessible and world travel feasible for all. The Journal on Active Aging, July/August 2018 17(5), pp.76-80.

Mimoto, A. (2009) Stationary Cycling through Google Streetview.

http://bako.ca/streetview-riding/index.html (retrieved 11 March 2019).

Raffman, J. (2009) The Nine Eyes of Google Street View. IMG MGMT, Art $F$ City. http://artfcity.com/2009/08/12/img-mgmt-the-nineeyes-of-google-street-view/ (retrieved 12 March 2019).

Raffman, J., and Steinman, K. (2016) John Rafman: Nine Eyes. New Documents, Los Angeles.

Smith, M. L. (2018) Riding Through Walls. In Douglas, S., \& Lowry, S. (eds.) Anywhere, 2. Project Anywhere, pp.104-106. 